\title{
叶片加工超差对高压压气机性能影响和 敏感性分析”
}

\author{
郑似玉 滕金芳 芫晓青 \\ (上海交通大学航空航天学院 上海 200240)
}

\begin{abstract}
摘要: 在航空发动机中, 高压压气机叶片的加工超差难以避免, 势必对压气机的气动性能造成影响。以某高压压气机后面级 作为研究对象, 采用数值模拟方法, 对典型的高压压气机加工超差种类进行敏感性分析。首先, 选取位置度、轮廓度及扭转 度三种典型加工超差, 采用高斯分布概率密度函数确定超差分布; 其次, 通过数值模拟, 研究加工超差对高压压气机等熵效 率、总压比等气动性能的影响规律, 提取气动性能对各类超差的敏感性参数。研究结果表明, 当叶型位置度变化时, 气动性 能基本都呈恶化趋势, 其中动叶根部轴向位置度某种变化情况使得喘振裕度减少 $25 \%$ 之多; 轮廓度与扭转度的变化与高压压 气机气动性能呈负线性的变化趋势; 在这三种典型超差类型中, 高压压气机气动性能对扭转度及轴向位置度超差的敏感性较 大，周向位置度和轮廓度次之。
\end{abstract}

关键词: 高压压气机; 加工超差; 敏感性分析; 气动性能

中图分类号: V231; TK474

\section{Sensitivity Analysis of Manufacturing Variability on High-pressure Compressor Performance}

\author{
ZHENG Siyu TENG Jinfang QIANG Xiaoqing \\ (School of Aeronautics and Astronautics, Shanghai Jiao Tong University, Shanghai 200240)
}

\begin{abstract}
Manufacturing variabilities of high-pressure compressor blades can hardly be avoided in aero-engines, which may seriously affect the aerodynamic performance of the compressors. To analyze the sensitivity of manufacturing variabilities on compressor performance, the rear stage of a high-pressure compressor is used to make the numerical simulation. Firstly, several typical manufacturing variabilities such as positional tolerance, profile tolerance and twisted tolerance are chosen and Gaussian distribution probability density function is used to determine the distribution of tolerance. Then the effective law of manufacturing variabilities to compressor's performance such as isentropic efficiency and total pressure ratio are investigated through numerical simulation, and the sensitivity parameters are extracted. The results indicate that when the position of blade changes, aerodynamic performance of high-pressure compressor are always reduced, for example, the axial position makes the surge margin decrease almost $25 \%$, while profile and twisted tolerance can lead to negative linear variation; throughout these three variabilities, the sensitivity of performance to the blade twist and axial position are larger, while circular position and profile tolerance have minor effects.
\end{abstract}

Key words: high-pressure compressor; manufacturing variability; sensitivity analysis; aerodynamic performance

\section{0 前言}

作为航空发动机的关键部件, 高压压气机对发 动机性能的影响至关重要。在高压压气机叶片的加 工过程中, 由于叶片弯扭形状的复杂, 以及数控铣、 锻造等不同加工工艺的作用, 使得加工超差难以避

* 航空基金资助项目(2015ZB57006)。20170318 收到初稿, 20170829 收到 修改稿
免, 各类加工超差(如扭转度、前尾缘形状、轮廓度、 位置度等)势必对压气机的气动性能造成影响。因 此, 深入研究压气机气动性能对不同超差的敏感性 大小, 对控制叶片加工精度、提高发动机性能具有 重要的意义。

国内外学者对典型的几种加工超差进行了较多 的研究。对于扭转度超差, 李晓丽等 ${ }^{[1]}$ 研究了叶片 安装角误差对整台压气机性能的影响, 发现叶片安 装角增大时气流的流通能力增强, 总压比和效率变 
化显著。LANGE 等 ${ }^{[2-4]}$ 通过数值模拟, 分析了动叶 安装角对高压压气机气动性能的影响, 他们的研究 结果表明, 第一级动叶安装角变化对折合流量的影 响最为显著, 而后面级的安装角变化的影响几乎可 以忽略。LEBELE-ALAWA 等 ${ }^{[5]}$ 研究了叶片扭转与 其耗功的关系, 发现叶型的扭转使气流折转角增加, 导致压气机耗功增加, 效率降低。对于前缘形状超 差, ROBERTS 等 ${ }^{[6]}$ 针对跨音速压气机动叶前缘部分 弦长变化的影响进行了试验研究, 试验表明弦长对 其性能基本无影响。SCHNELL 等 ${ }^{[7-8]}$ 针对风扇叶片, 进行了前缘厚度、弦长、前缘角及前缘平头等不同 前缘形状影响的研究, 通过比较各种情况下性能的 标准差发现, 前缘厚度及前缘角度对气动性能参数 的影响较为显著。对于轮廓度超差, 加工、积垢、 涂层等多个因素皆会造成轮廓度变化。王松等 ${ }^{[9-10]}$ 从叶片积垢导致的轮廓度变化着手, 发现其会导致 吸力面中部压力梯度较大区域提前转捩, 增加叶栅 的流动损失。ELMSTROM 等 ${ }^{[11]}$ 从不均匀涂层导致 的前缘轮廓度变化着手, 发现其会造成叶片前端压 力梯度变化, 产生分离泡。张伟吴等 ${ }^{[12]}$ 针对浴轮叶 型加工产生的轮廓度偏差, 采用非定常数值模拟结 合整机试验的方法进行研究, 结果表明, 通道浴和 泄漏流的发展、激波的形态和强度等流动结构等都 会显著改变。对于位置度超差, 相当于叶片的轻微 弯掠变形, 而今, 研究集中于尺度较大的弯掠变化。 BENINI ${ }^{[13]}$ 以 NASA Rotor 37 动叶为原型进行了周 向弯和轴向掠的改型研究, 发现弯和掠会影响激波 和二次流的结构, 且优化叶片弯和掠的程度可使压 气机效率有 $1.3 \%$ 的提高。陈浮等 ${ }^{[14-15]}$ 通过研究压气 机叶片的弯掠变形发现优化的弯掠叶片能改善流场 结构, 并降低二次流损失。

近年来, 针对多类超差的综合研究及敏感性分 析逐渐出现。FATHI 等 ${ }^{[16]}$ 对涡轮叶片的扭转度、位 置度、前尾缘厚度及轮廓度这四种类型进行了敏感 性分析, 结果表明扭转度和轮廓度对效率及折合流 量影响最为显著。WUNSCH 等 ${ }^{[17]}$ 将叶尖间隙、前 尾缘角度这几类参数作为超差变量进行了不确定性 研究, 结果表明前缘角度的变化对压气机性能的影 响最明显。

综上所述, 国内外学者对单类加工超差的研 究已较为普遍, 但有关性能对加工超差的敏感性 研究还较为稀少, 处于起步阶段, 值得我们深入 研究。在加工中, 各类超差难以避免, 而通过敏 感性分析则可篮选出最为关键的超差因素进行重 点关注, 减小其对性能的影响程度。基于此, 本 文将以某高压压气机的后面级为研究对象, 选取
轮廓度、位置度及扭转度这三类典型超差, 通过 数值模拟手段, 详细研究不同加工超差对压气机 等熵效率、总压比等气动性能参数的影响, 并进 行参数的敏感性分析，从而篎选出对高压压气机 气动性能影响较大的超差类型, 为航空发动机叶 片高精度制造提供依据。

\section{1 研究对象及数值方法}

本文的研究对象为某多级高压压气机的后面级 (相关几何参数见表 1), 采用 Numeca Fine/Turbo 软 件对其进行数值研究。数值计算时采用 Spalart-Allmaras 一方程湍流模型(SA 湍流模型), 空 间离散采用中心差分格式, 时间推进采用四阶 Runge-Kutta 方法加速迭代求解，进口给定总温、总 压及速度分布, 出口按径向平衡方程给定平均静压, 动静交界面采用一维无反射边界条件。

\section{表 1 基本几何参数}

\begin{tabular}{cc}
\hline 几何参数 & 数值 \\
\hline 进口轮勃比 & 0.913 \\
出口轮毫比 & 0.926 \\
动叶展弦比 & 1.71 \\
动叶稠度 & 1.233 \\
静叶展弦比 & 1.10 \\
静叶稠度 & 1.798 \\
\hline
\end{tabular}

为验证本文所采用的数值方法的准确性, 利用 具有试验数据的相似几何进行数值校核。由于 NACA65 系列叶型具有丰富的试验数据, 且其叶型 设计符合高压压气机出口级, 即本文研究对象的特 点, 因此, 综合考虑之下, 数值验证的几何模型采 用 NACA65-(12)10, 其几何示意图及计算网格如图 $1 \mathrm{a}$ 所示。图 $1 \mathrm{~b}$ 为零攻角、进口马赫数约为 0.69 的 工况下数值模拟结果与试验结果得到的静压系数 分布的对比, 由图 $1 \mathrm{~b}$ 可见, SA 湍流模型下得到的 数值结果与试验结果的吻合情况基本较好, 其中, 在吸力面上, 试验值轻微偏高, 最大偏差约为 $11.85 \%$, 证明本课题中采用的数值方法具有较高的 准确性。

在使用计算流体力学 (Computational fluid dynamics, CFD)方法进行数值模拟时, 存在一定的 局限性, 必将会产生一定的计算误差 ${ }^{[18]}$, 而由于超 差本身的几何变化范围很小，因此其计算得到的结 果是源于计算误差还是几何超差的影响就变得很难 判断。因此, 在本文中, 所有的参数设置都与原型 相同, 仅比较超差几何相对于原型的性能变化程度, 一定程度上避免了 CFD 中的计算误差问题。 


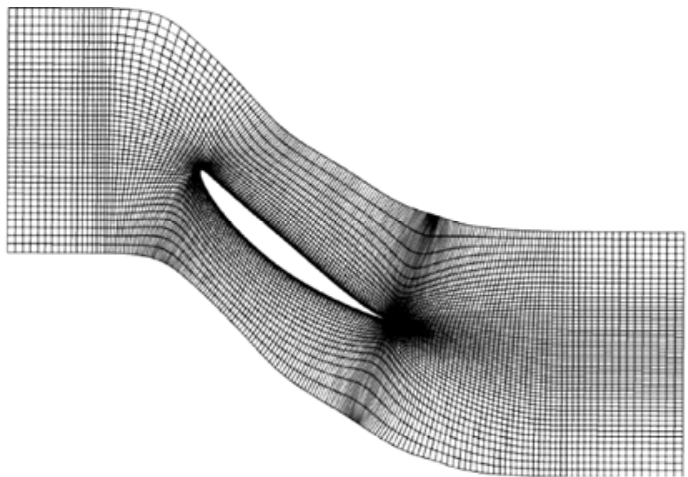

(a) NACA65-(12)10 翼型几何及网格

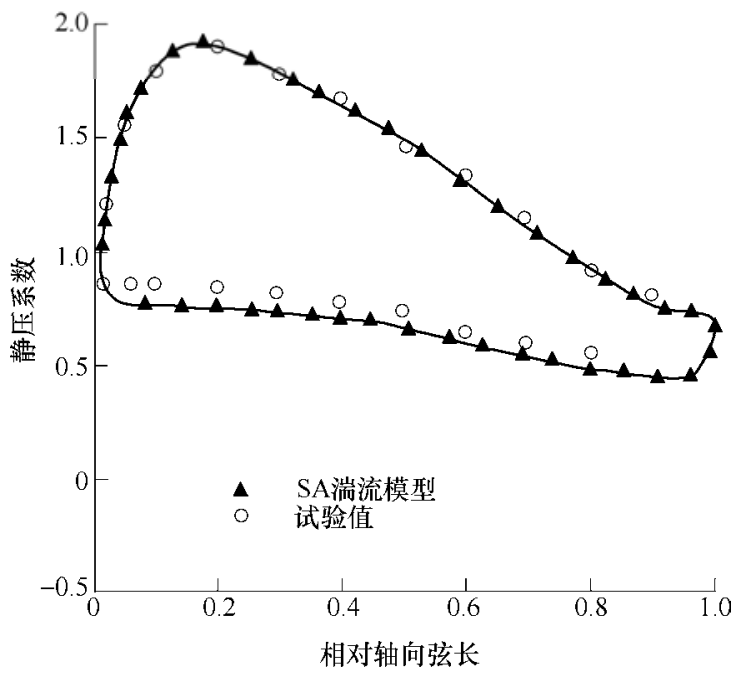

(b) 数值模拟与试验结果的静压系数对比

图 1 数值验证的几何与结果

计算网格的三维及二维示意图分别由图 2a 及 图 2b 所示, 该网格采用 $\mathrm{O} 4 \mathrm{H}$ 型拓扑结构, 壁面第 一层网格厚度设定为 $5 \times 10^{-6} \mathrm{~m}$, 以保证近壁面处边 界层内具有合理的网格布置, 从而获得高质量的壁 面边界层数值结果。同时, 对网格无关性进行了研 究, 具体网格配置如表 2 所示。由表 2 中的数据可

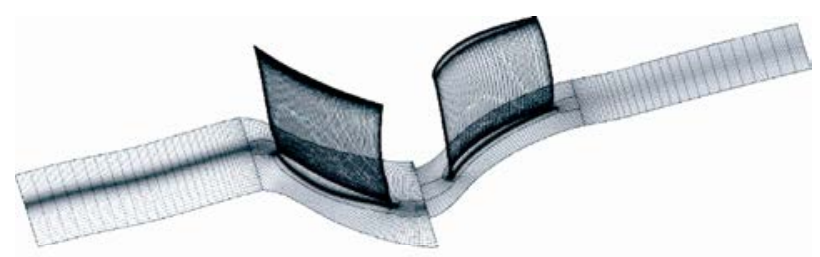

(a)三维网格示意图

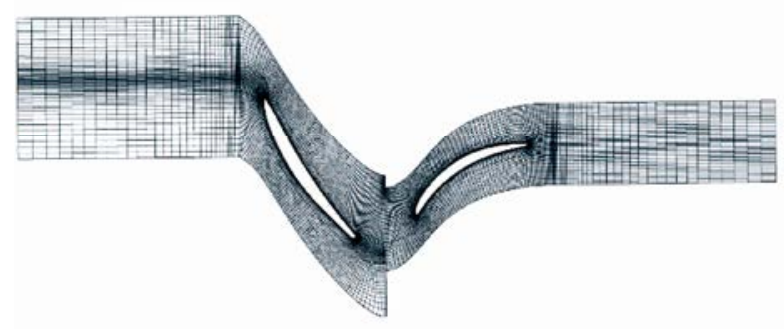

(b) 二维网格示意图

图 2 研究对象计算网格示意图
见, 当网格数达到 185 万以上时, 效率及总压比的 变化量几乎为 0 , 所以选定 185 万网格进行后续的 研究。

表 2 网格无关性分析

\begin{tabular}{ccccccc}
\hline $\begin{array}{c}\text { 序 } \\
\text { 号 }\end{array}$ & 网格数/万 & $\begin{array}{c}\text { 等熵 } \\
\text { 效率 }\end{array}$ & $\begin{array}{c}\text { 效率变化量 } \\
(\%)\end{array}$ & 总压比 & $\begin{array}{c}\text { 折合 } \\
\text { 流量 }\end{array}$ & $\begin{array}{c}\text { 流量变化量 } \\
(\%)\end{array}$ \\
\hline 1 & 73 & 0.8383 & 0 & 1.203 & 1.0249 & 0 \\
2 & 112 & 0.8397 & 0.167 & 1.203 & 1.0254 & 0.049 \\
3 & 145 & 0.8407 & 0.286 & 1.204 & 1.0259 & 0.098 \\
4 & 185 & 0.8409 & 0.310 & 1.204 & 1.0268 & 0.185 \\
5 & 224 & 0.8410 & 0.320 & 1.204 & 1.0268 & 0.185 \\
\hline
\end{tabular}

\section{2 超差分布及敏感性分析}

根据前文分析, 选取位置度、轮廓度及扭转度 这三个典型加工超差进行详细研究。其中, 位置度 可分为周向位置度及轴向位置度两种, 分别改变指 定截面叶型的周向坐标或轴向坐标, 完成位置度超 差几何构造; 轮廓度超差可通过 NUMECA 中 IGG 模块的叶型偏置功能进行几何改造; 扭转度则通过 IGG 模块的旋转功能将叶型绕其重心旋转一定的角 度。各超差几何变化的示意图如图 3 所示, 其中, 实线表示原型几何的叶型, 虚线代表几何超差范围 的边界。

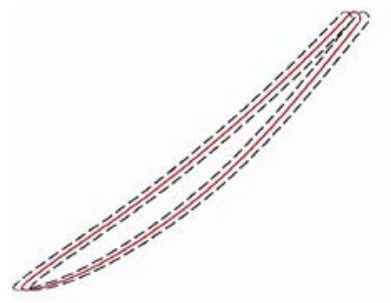

(a) 周围位置度

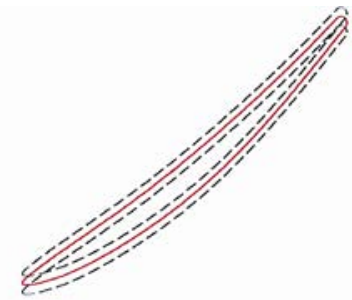

(b) 轴向位置度

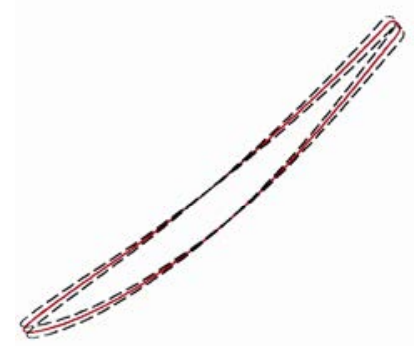

(c) 扭转度

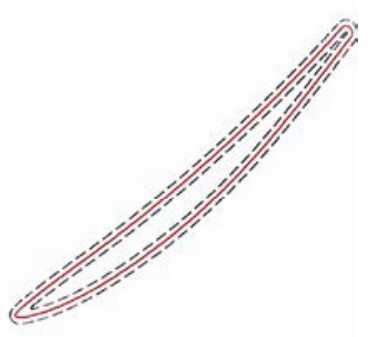

(d) 轮廊度
图 3 各超差几何变化示意图

对于所研究的高压压气机级, 在其制造检测过 程中, 以动、静叶中高度分别为 $20 \%$ 、 $50 \%$ 及 $70 \%$ 叶高的三个截面作为检测截面进行质量检测, 因此 在本文中, 将采取动、静叶的上述各三个检测截面 作为具体超差变化对象。根据不同超差类型分别 单独改变各个截面的几何参数, 用于网格重构以 
进行数值模拟和敏感性分析, 根据加工经验及航 标中对误差范围的规定, 规定各类超差取值范围 如表 3 所示。

表 3 超差类型及取值范围

\begin{tabular}{cccc}
\hline 超差类型 & $\begin{array}{c}\text { 标准值 } \\
\text { (相对于原型) }\end{array}$ & 变化范围 & $\begin{array}{c}\text { 变化步长 } \\
\Delta x\end{array}$ \\
\hline 周向位置度 $/ \mathrm{mm}$ & 0 & $-1 \sim 1$ & 0.5 \\
轴向位置度 $/ \mathrm{mm}$ & 0 & $-1 \sim 1$ & 0.5 \\
轮廓度 $/ \mathrm{mm}$ & 0 & $-0.1 \sim 0.1$ & 0.05 \\
扭转度 $/\left(^{\circ}\right)$ & 0 & $-1 \sim 1$ & 0.5 \\
\hline
\end{tabular}

对上述超差类型进行详细的数值模拟后, 对计 算结果进行误差和敏感性分析。在假定几何超差分 布满足高斯分布概率密度函数的前提下, 当超差大 小分布在不同区域时, 对应的概率大小也会随之变 化。高斯分布概率密度函数的关系式如式(1)所示

$$
f(x)=\frac{1}{\sigma \sqrt{2 \pi}} \exp \left(-\frac{(x-\mu)^{2}}{2 \sigma^{2}}\right)
$$

式中, $\mu$ 为输入几何参数相对原型变化量的平均值, $\sigma$ 为标准差。

当 $\mu=0 、 \sigma=0.3$ 时, 对应的高斯分布概率密度 函数的图像如图 4 所示。

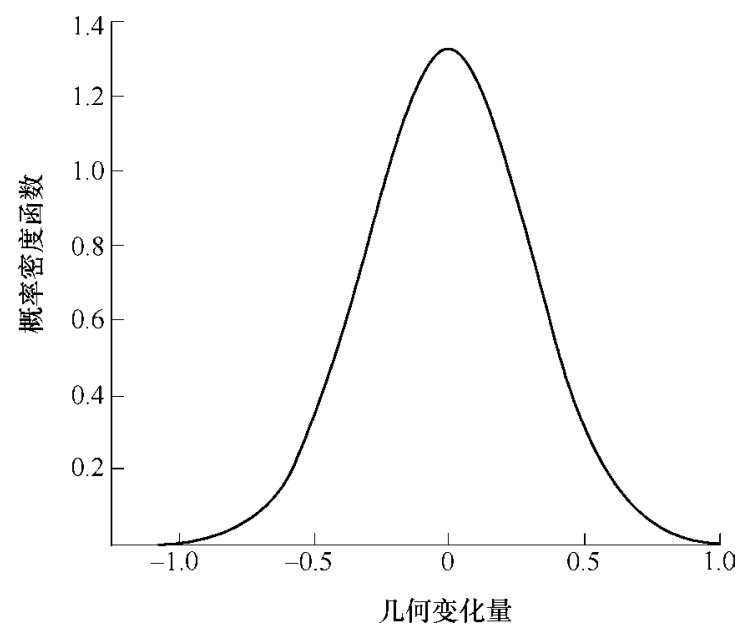

图 4 高斯分布概率密度函数

输出性能参数的平均值、均方差、标准差以及 敏感性参数则可根据式(2) (5)求得

平均值

$$
\mu_{\varphi}=\sum_{i=1}^{N_{p}} \omega_{i} \varphi_{i}
$$

均方差

$$
\sigma_{\varphi}^{2}=\sum_{i=1}^{N_{p}} \omega_{i}\left(\varphi_{i}^{2}-\mu_{\varphi}^{2}\right)
$$

标准差

$$
\sigma_{\varphi}=\sqrt{\sum_{i=1}^{N_{p}} \omega_{i}\left(\varphi_{i}^{2}-\mu_{\varphi}^{2}\right)}
$$

敏感性参数

$$
s_{\varphi}=\sum_{i=1}^{N_{p}} \omega_{i}\left|\frac{\Delta \varphi}{\Delta x}\right|
$$

式中, $\omega_{i}$ 为输入几何参数的概率值, $\varphi_{i}$ 为对应输出 性能参数值的大小, $N_{p}$ 为总的配置点数, $\Delta \varphi$ 为相对 于原型的性能参数变化量, $\Delta x$ 为超差参数变化步长。

\section{3 计算结果及分析}

根据第 1 部分确定的网格和数值方法, 分别对 三种超差类型对应的几何方案进行了详细的数值模 拟，下面进行具体的分析和探讨。

由所有方案的数值模拟结果显示, 在所研究的 动、静叶共 6 个制造截面中, 各截面加工超差所导 致的影响程度各不相同, 考虑到篇幅的限制, 仅选 择影响相对较大的截面的几何超差进行具体分析。

\section{1 位置度的影响}

就位置度超差而言, 轴向位置度对高压压气机 级性能的影响程度明显高于周向位置度, 其中, 又 以动叶根部轴向位置度的影响为最, 以其为例进行 分析讨论。

图 5 给出了轴向位置度超差对高压压气机级总 体性能参数的影响规律。图 5 中, 散点为不同几何 计算得到的性能参数点, 黑色实线为经过不确定性 分析(uncertainty quantification, UQ)处理得到的气动 性能平均值, 并给出了 $\pm 3 \sigma$ 的误差带(在 $\pm 3 \sigma$ 的误差 分布下，置信区间可达 99.73\%), 基本可以涵盖所 有几何偏差下的性能点。

如图 5 所示, 随着轴向位置度的变化, 高压压 气机级的等熵效率和总压比都呈现逐步恶化的趋 势, 且轴向位置度相对原型变化愈大，等熵效率下 降愈明显。当轴向位置度为负时导致的性能恶化程 度相比正方向更为严重。以设计点(0.99流量工况点) 为例, 当轴向位置度偏差达到 $-1 \mathrm{~mm}$ 时, 压气机的 等熵效率下降了约 $0.45 \%$ ，总压比下降了约 $0.32 \%$; 而当偏差为 $+1 \mathrm{~mm}$ 时, 等熵效率下降 $0.14 \%$, 总压 比下降 $0.09 \%$ 。另外, 由轴向位置度变化 $-1 \mathrm{~mm}$ 后 的压气机特性线可见，高压压气机的近喘点流量相 对于其他几种方案发生了明显变化, 近喘点流量由 0.91 增大至 0.96 , 其稳定工作的范围相比其他方案 大幅度减小，喘振裕度减小了约 $25 \%$ 。并且，与周 向位置度超差相比, 轴向位置度方案下的误差分布 带范围更宽, 可见其导致的性能恶化情况更为显著。 


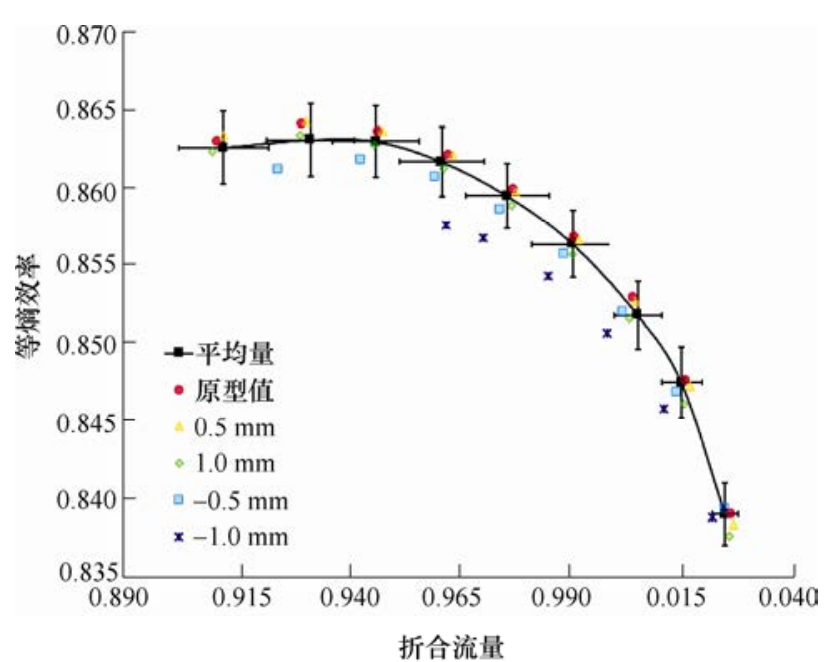

(a) 等摘效率

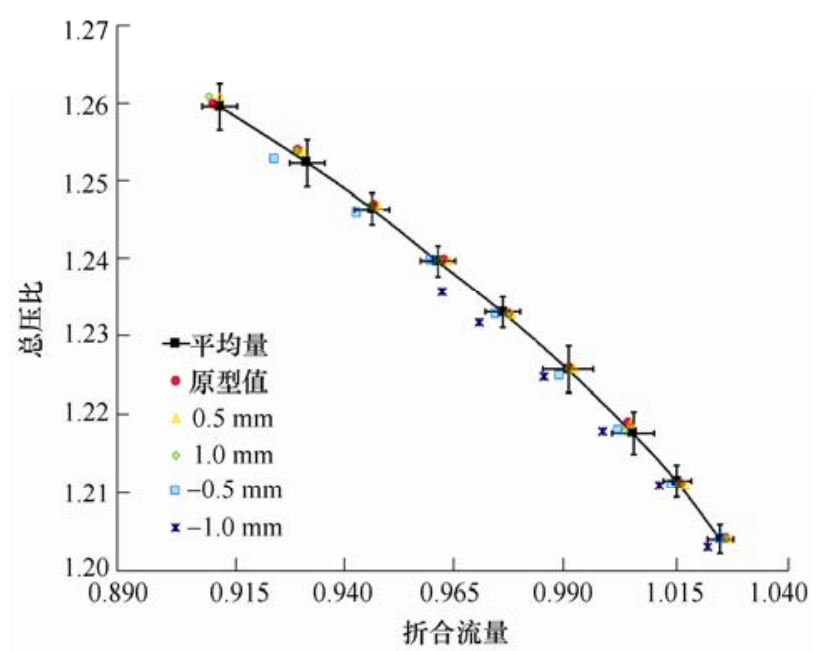

(b) 总压比

图 5 轴向位置度超差对压气机总体性能的影响(动叶根部)

当根部截面轴向位置度增大或减小时, 叶片根 部发生轻微前掠或后掠, 从而对流场产生微小扰动。 图 6 给出了动叶 $20 \%$ 叶高截面不同轴向位置度情况 下该截面静压系数的对比。观察静压系数分布发现,

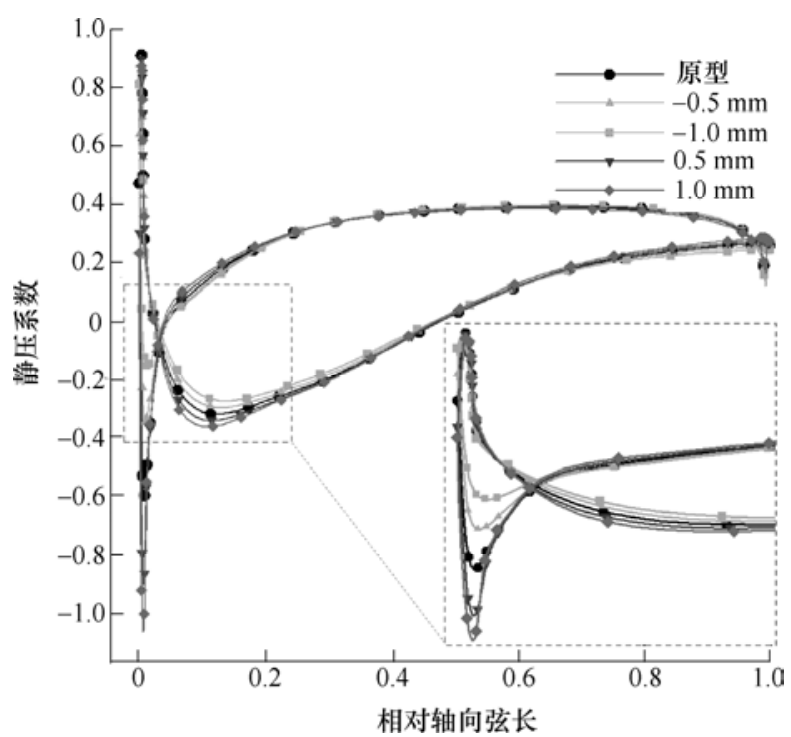

图 6 轴向位置度超差下动叶 $20 \%$ 叶高静压系数
轴向位置度超差致使动叶前半部分和后半部分的气 动载荷发生不同趋势的增减，同时，导致前缘附近 的逆压梯度以及进口攻角的变化。在轴向位置度变 化 $-1 \mathrm{~mm}$ 的情况下, 其性能恶化最为显著, 观察图 7 动叶出口位置的总压损失系数展向分布图可见， 当根部轴向位置度变化 $-1 \mathrm{~mm}$, 在 $20 \%$ 叶高附近处 的总压损失系数相对于原型增大了近 $20.8 \%$, 可见 其损失之多。

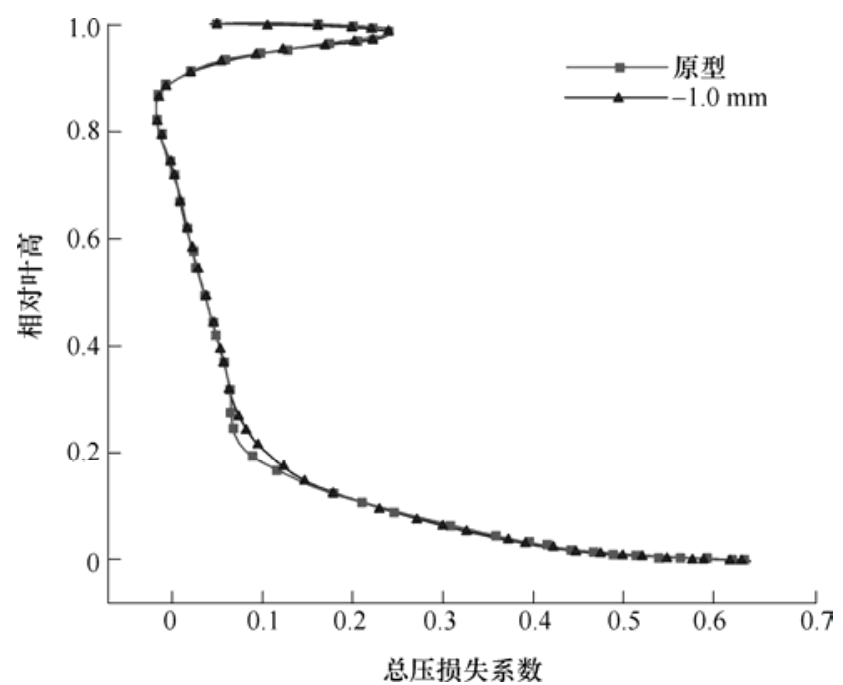

图 7 动叶出口总压损失系数展向分布对比图

\section{2 轮廓度的影响}

就轮廓度的影响而言, 动叶尖部轮廓度的变化 对高压压气机级性能的影响较为显著, 图 8 给出了 尖部截面轮廓度超差对高压压气机级总体性能参数 的影响规律。由图 8 可见, 随着轮廓度向外增加, 压气机级的等熵效率及总压比逐渐下降，当偏置量 达到 $+0.1 \mathrm{~mm}$ 时，在设计工况下，等熵效率下降了 约 $0.144 \%$, 总压比下降了约 $0.148 \%$ 。而当轮廓度 向内偏置时，压气机级的等熵效率及总压比逐渐上 升，当偏置量达到 $-0.1 \mathrm{~mm}$ 时，等熵效率上升了约 $0.027 \%$, 总压比上升了约 $0.015 \%$ 。由此可见, 轮廓 度减小有利于提高压气机级性能, 但其收益较小, 相比轮廓度增大时的性能恶化降低了一个数量级。 因此, 制定叶片加工公差时, 需要适当减小正向轮 廓度取值范围, 以减少压气机性能恶化情况的发生。

由于轮廓度的增大, 流道的喉道面积减小, 使 得压气机的通流能力有所减小; 同时，其也会导致 叶片前缘钝度增大，前缘处的损失随之增加，因此 轮廓度外置 $0.1 \mathrm{~mm}$ 时, 其性能恶化相对显著。由图 9 给出的动叶 $70 \%$ 叶高截面不同轮廓度下该截面静 压系数的对比可见, 轮廓度愈大, 进口攻角减小, 使得进口负攻角情况愈严重, 造成的前缘附近的流 动损失也愈大。图 10 为动叶 $70 \%$ 叶高平面出口位 
置的相对轴向速度随截距位置变化的曲线图, 其中, 相对轴向速度突变段的横轴宽度可代表尾迹宽度, 纵轴长度可代表尾迹强度。由图 10 可见, 轮廓度变 化 $0.1 \mathrm{~mm}$ 的情况尾迹强度显著大于原型, 其尾迹损 失严重。

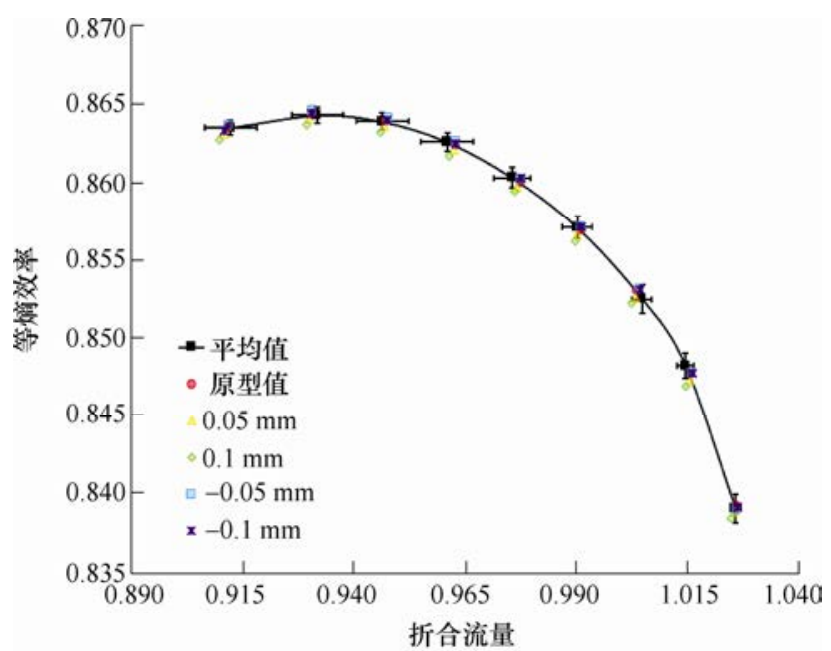

(a) 等摘效率

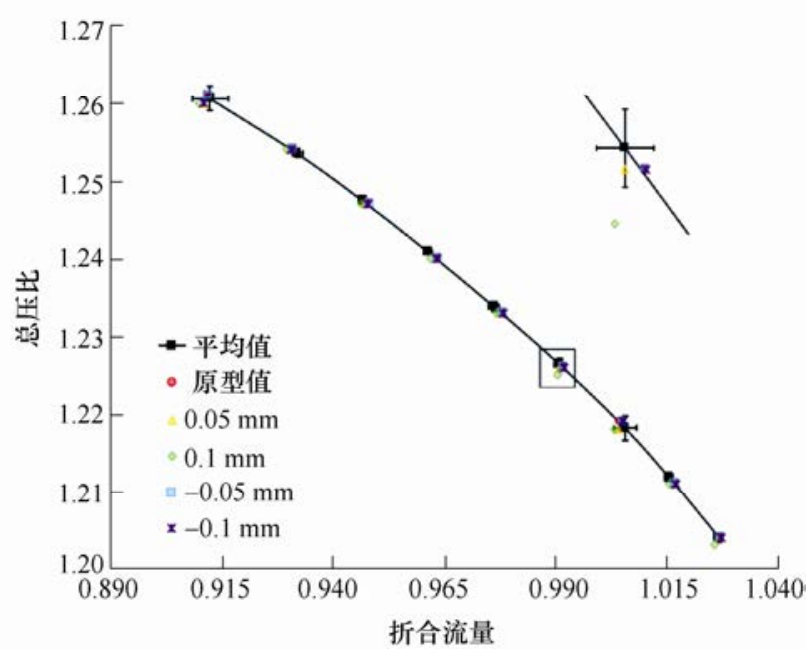

(b) 总压比

图 8 轮廓度超差对压气机总体性能的影响(动叶尖部)

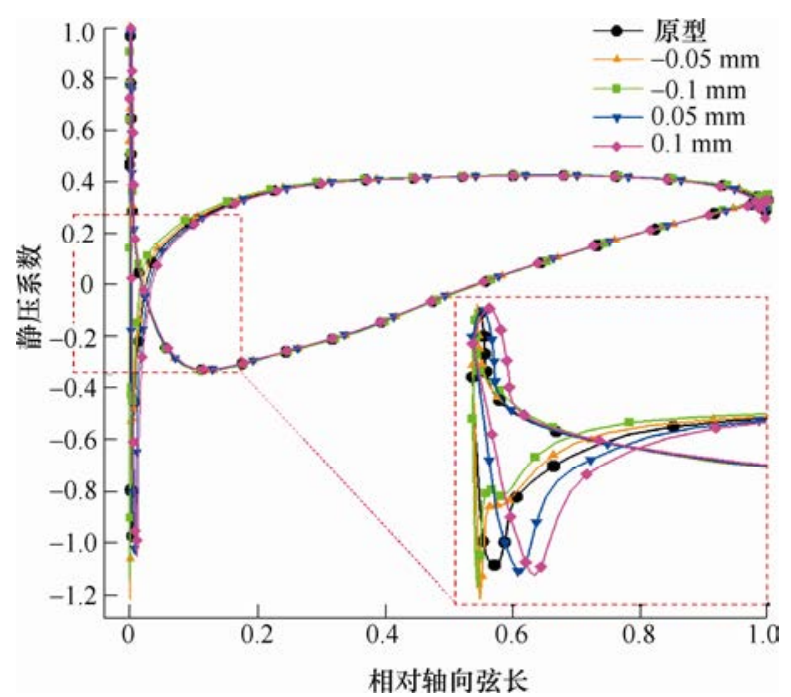

图 9 轮廓度超差下动叶 $70 \%$ 叶高静压系数

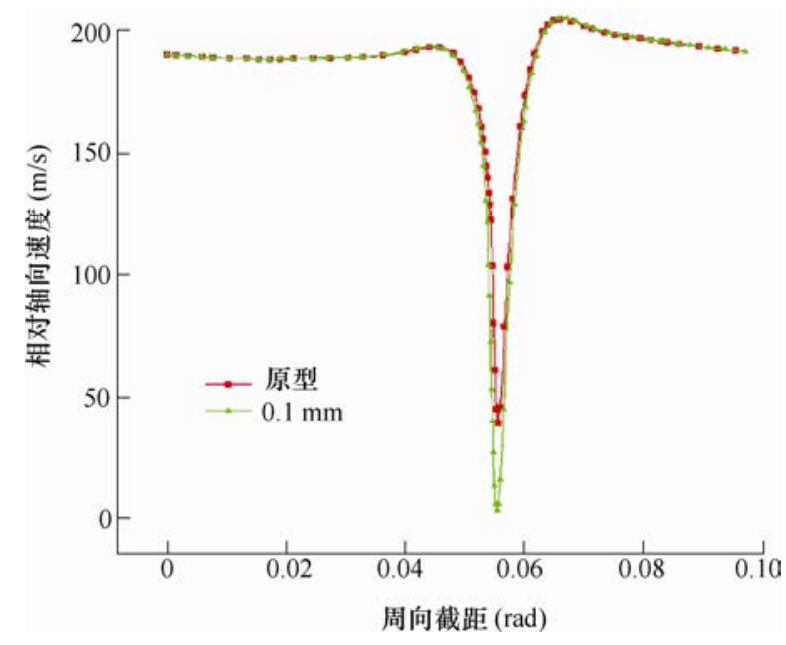

图 10 动叶 70\%叶高出口位置相对轴向速度分布

\section{3 扭转度的影响}

对于扭转度超差, 动叶尖部的影响同样较为显 著, 图 11 给出了尖部截面扭转度超差对压气机级等 熵效率及总压比特性的影响规律。根据定义, 当动 叶绕重心旋转角度为正时, 安装角减小; 相反, 旋 转角度为负时, 安装角增大。

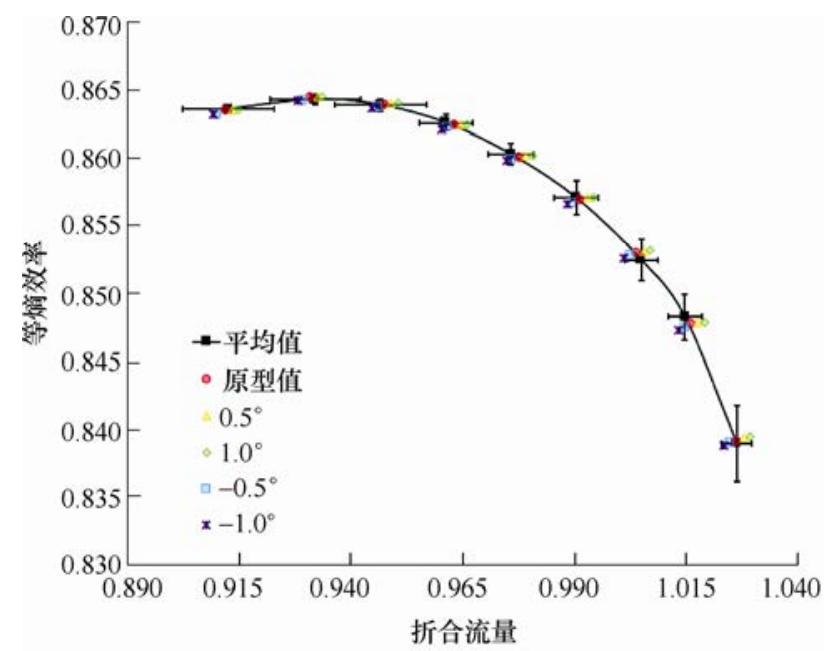

(a) 等摘效率

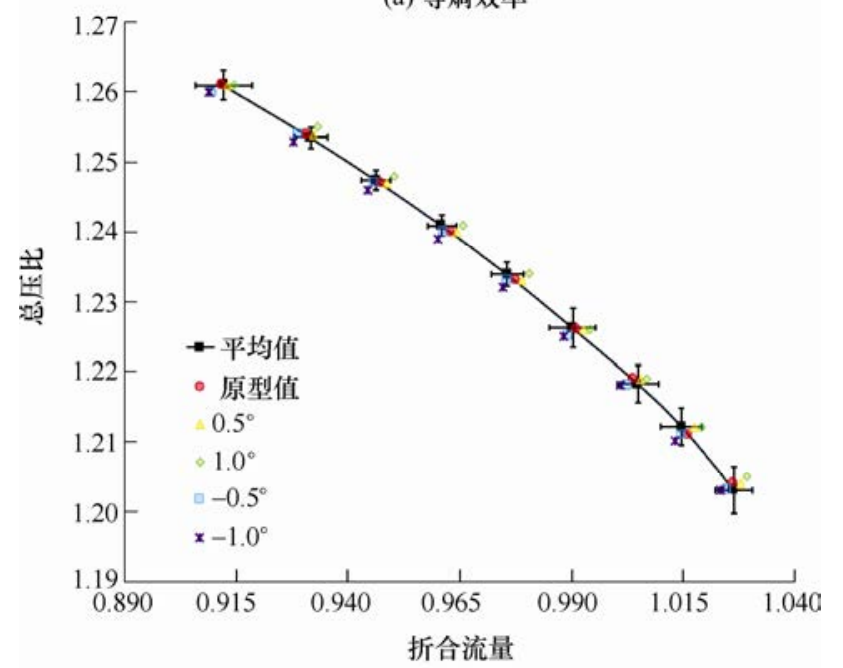

(b) 总压比

图 11 扭转度超差对压气机总体性能的影响(动叶尖部) 
如图 11 所示, 当动叶叶尖发生扭转时, 压气机 级等熵效率及总压比的变化情况与扭转度变化量基 本呈线性关系。即, 安装角增大时, 动叶叶尖附近 的进口负攻角增大, 损失增加从而导致级性能下降。 在设计工况下, 当安装角增大 $1^{\circ}$ 时 (在图 11 中即为 旋转 $\left.-1^{\circ}\right)$, 压气机级的等熵效率减小了 $0.15 \%$, 总 压比减小了 $0.214 \%$; 反之, 当安装角减小时, 压气 机级的等熵效率和总压比逐渐增大。另外, 通过误 差带分布可知, 近堵工况下的性能变化量远大于近 喘点工况, 由此可见, 扭转度超差对压气机性能的 影响程度与压气机的工作条件也有极大的关联。

图 12 为不同扭转度超差分布下动叶 $70 \%$ 叶高 处的静压系数对比图。由图 12 可见, 在前缘附近, 随着旋转角度的减小, 即扭转度的增大, 动叶压力 面侧静压系数增大, 吸力面侧静压系数减小, 意味 着前缘附近的叶片加功量增大, 叶片载荷增加。另 外, 随着扭转度的增大, 进口攻角减小, 直接导致 叶片进口负攻角加剧, 由此造成的流动损失增多, 这也一定程度上解释了扭转度增大导致压气机等熵 效率及总压比下降的原因。图 13 的熵云图对比更直 观地反映了前缘附近的损失变化, 扭转度增大 $1^{\circ}$ 时, 前缘处压力面有明显的熵增, 可见其损失更为 严重。

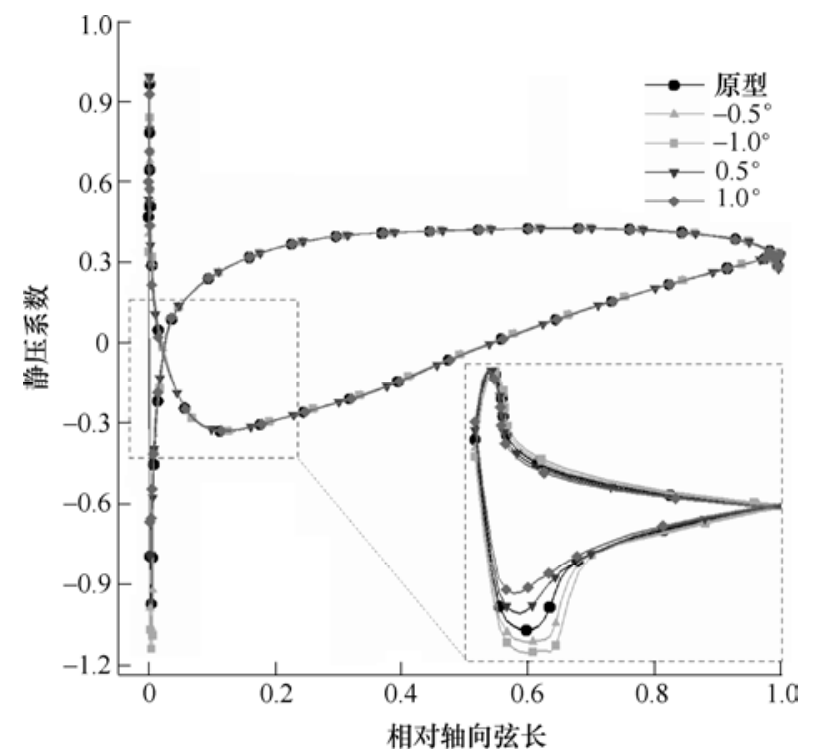

图 12 扭转度超差下动叶 $70 \%$ 叶高静压系数

\section{4 敏感性分析}

根据式(5)计算各类超差的敏感性参数, 以此来 分析压气机性能对于不同超差类型的敏感性大小, 从而反映当几何产生偏差时压气机性能偏离原型值 的程度, 也凭此篮选出对总体性能影响程度相对较 大的超差类型, 以指导加工及后续维修过程中几何 超差范围的选取。

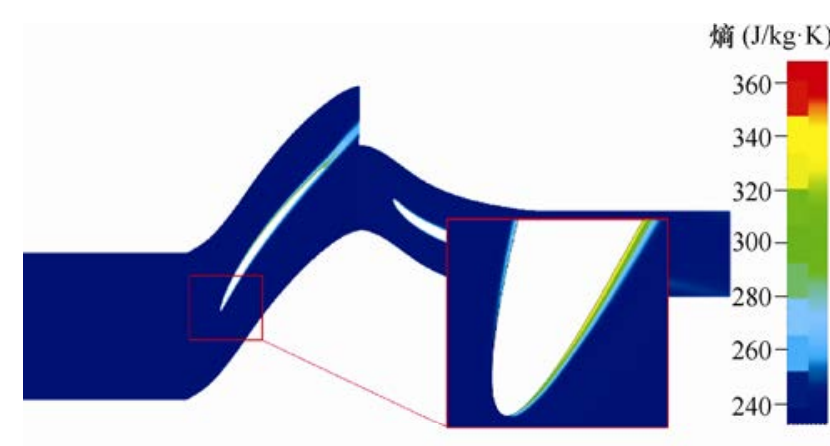

(a) 原型

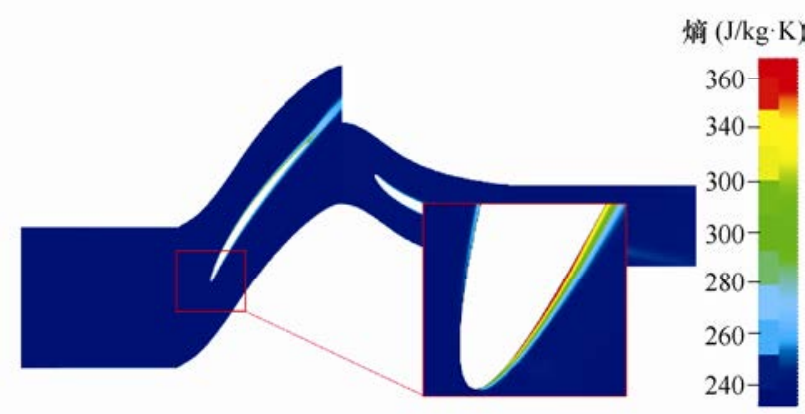

(b) 扭转度增大 $1^{\circ}$

图 13 动叶 $70 \%$ 叶高截面熵云图对比

图 14 给出了设计工况下, 等熵效率及总压比的 敏感性参数柱状图, 其中 S1、S2、S3 分别指代动 叶的根、中、尖截面, S4、S5、S6 分别指代静叶的 根、中、尖截面。由图 14a 可见, 高压压气机级的 等熵效率分别对动叶尖部扭转度、轮廓度以及动叶

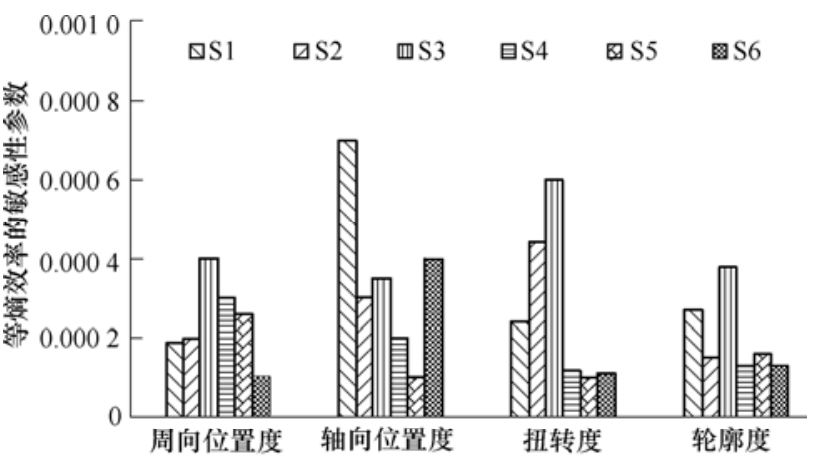

(a) 等摘效率对超差的敏感性参数

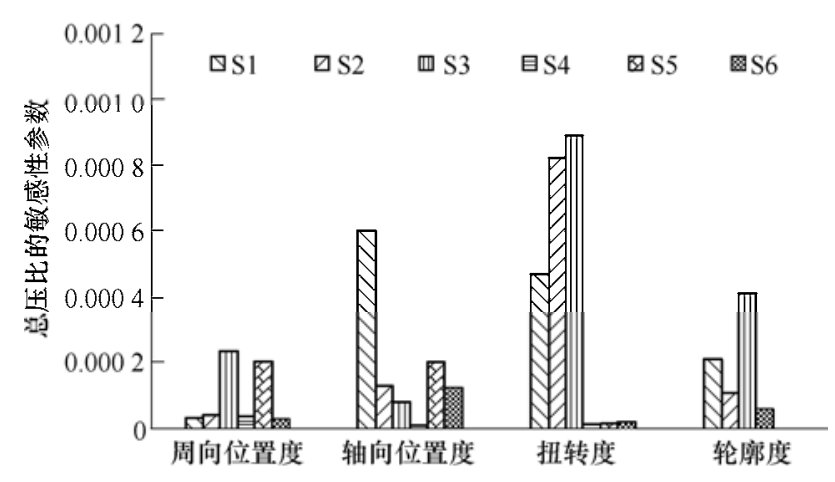

(b) 总压比对超差的敏感性参数

图 14 压气机性能参数对加工超差的敏感性分析 
根部轴向位置度超差的敏感性最强; 而由图 14b 可 见, 压气机级的总压比对动叶尖部及中部截面的扭 转度以及动叶根部的轴向位置度超差的敏感性最 强。总体看来, 压气机级总体性能对动叶加工超差 的敏感性远大于静叶; 其中, 又以动叶的扭转度及 轴向位置度影响最甚。

\section{4 结论}

本文以某多级高压压气机的后面级为研究对 象, 采用定常数值模拟和基于概率密度函数的理论 分析方法, 对典型叶片加工超差类型进行了详细的 计算和分析, 得到了如下结论。

(1) 几何超差对高压压气机级性能的影响各不 相同: 当叶片的位置度超差变化时, 高压压气机的 气动性能呈现恶化趋势, 其中, 轴向位置度变化 -1 $\mathrm{mm}$ 的案例使得喘振裕度减小了 $25 \%$ 之多, 对压气 机的稳定工作影响极大, 在加工中尤需要注意和避 免; 当叶片的轮廓度与扭转度变化时, 高压压气机 的气动性能随几何的改变基本呈负线性的变化趋 势, 即轮廓度或扭转度增大, 导致压气机性能恶化。 因此, 建议尽量减小轮廓度与扭转度正向变化的取 值范围。

（2）敏感性分析有助于篮选出对总体性能影响 程度相对较大的超差类型, 以指导加工及后续维修 过程中几何超差范围的选取。分析结果表明, 在位 置度、轮廓度及扭转度这三种典型超差中, 高压压 气机级的气动性能对动叶各截面的扭转度及轴向位 置度超差的敏感性较强, 周向位置度和轮廓度次之; 静叶各截面几何超差的影响则较不显著。因此, 在 实际叶片加工中, 需要着重关注动叶中的扭转度及 轴向位置度这两种超差类型, 避免较大的加工误差 出现导致压气机性能的较大偏离。

\section{参 考 文 献}

[1] 李晓丽, 楚武利. 安装角变化对多级轴流压缩机性能影 响的分析[J]. 风机技术, 2008 (5): 27-29.

LI Xiaoli, CHU Wuli. Analysis on the influence of variable installation angle on performance of multi-stage axial-flow compressor[J]. Compressor, Blower and Fan Technology, 2008(5): 27-29.

[2] LANGE A, VOIGT M, VOGELER K, et al. Impact of manufacturing variability on multistage high-pressure compressor performance[J]. Journal of Engineering for Gas Turbines and Power, 2012, 134(11): 417-426.

[3] LANGE A, VOIGT M, VOGELER K, et al. Probabilistic
CFD simulation of a high-pressure compressor stage taking manufacturing variability into account[C]// American Society of Mechanical Engineers. Proceedings of ASME Turbo Expo 2010, Power for Land, Sea, and Air, June 14-18, 2010, Glasgow, Brussels UK. ASME, 2010: 617-628.

[4] MARX J, STÄDING J, REITZ G, et al. Investigation and analysis of deterioration in high pressure compressors due to operation[J]. CEAS Aeronautical Journal, 2014, 5(4): 515-525.

[5] LEBELE-ALAWA B T, HART H I, OGAJI S O T, et al. Rotor-blades' profile influence on a gas-turbine's compressor effectiveness[J]. Applied Energy, 2008(85): 494-505.

[6] ROBERTS W B, ARMIN A, KASSASEYA G, et al. The effect of variable chord length on transonic axial rotor performance[J]. Journal of Turbomachinery, 2002 , 124(3): 351-357.

[7] SCHNELL R, KAMPMANN T L, NICKE E. On the impact of geometric variability on fan aerodynamic performance, unsteady blade row interaction, and its mechanical characteristics[J]. Journal of Turbomachinery, 2014, 136(9): 091005.

[8] GIEBMANNS A, SCHNELL R, STEINERT W, et al. Analyzing and optimizing geometrically degraded transonic fan blades by means of 2D and 3D simulations and cascade measurements[C]// American Society of Mechanical Engineers. Proceedings of ASME Turbo Expo 2012. Copenhagen: ASME, 2012: 279-288.

[9] 王松, 王国辉, 韩青, 等. 叶片积垢对压气机性能衰退 的影响 [J]. 哈尔滨工程大学学报, 2014, 35(12): 1524-1528.

WANG Song, WANG Guohui, HAN Qing, et al. Compressor performance deterioration caused by blade fouling[J]. Journal of Harbin Engineering University, 2014, 35(12): 1524-1528.

[10] 李钊, 王永华, 黄帅, 等. 积垢对压气机流场影响的数 值分析 [J]. 海军航空工程学院学报, 2011, 26(6): 637-644.

LI Zhao, WANG Yonghua, HUANG Shuai, et al. Numerical analysis of the effect of fouling on compressor flow field[J]. Journal of Naval Aeronautical and Astronautical University, 2011, 26(6): 637-644.

[11] ELMSTROM M E, MILLSAPS K T, HOBSON G V, et al. Impact of nonuniform leading edge coatings on the aerodynamic performance of compressor airfoils[J]. Journal of Turbomachinery, 2011， 133(4): 041004.

[12] 张伟昊, 邹正平, 刘火星, 等. 叶型偏差对整机环境中 
浴轮性能的影响 $[\mathrm{J}]$. 工程热物理学报, 2010, 31(11): 1830-1834.

ZHANG Weihao, ZOU Zhengping, LIU Huoxing, et al. Effects of profile deviation on turbine performance in whole engine environment[J]. Journal of Engineering Thermophysics, 2010, 31(11): 1830-1834.

[13] BENINI E, BIOLLO R. Aerodynamics of swept and leaned transonic compressor-rotors[J]. Applied Energy, 2007, 84(10): 1012-1027.

[14] 陈浮, 赵桂杰, 宋彦萍, 等. 叶片弯、掠对压气机端壁 流动的影响[J]. 工程热物理学报, 2004, 25(2): 211-215. CHEN Fu, ZHAO Guijie, SONG Yanping, et al. Influences of swept and curved blade on the flow near compressor cascade endwall[J]. Journal of Engineering Thermophysics, 2004, 25(2): 211-215.

[15] 冯秀莲, 金东海, 桂幸民. 叶片弯掠对压气机静子叶片 气动性能影响的三维数值模拟 [J]. 航空动力学报, 2009, 24(10): 2338-2343.

FENG Xiulian, JIN Donghai, GUI Xingmin. Numerical Simulation of the aerodynamics performance of swept and curved blade on the compressor stator[J]. Journal of Aerospace Power, 2009, 24(10): 2338-2343.

[16] FATHI A, ALIZADEH M. Effects of blade manufacturing deviations on turbine performance[C]// American Society of Mechanical Engineers. Proceedings of the ASME 2012 Gas Turbine India Conference, December 1, 2012, Mumbai, Maharashtra, India. Gurgaon: ASME, 2012: 203-211.

[17] WUNSCH D, HIRSCH C, NIGRO R, et al. Quantification of combined operational and geometrical uncertainties in turbo-machinery design[C]// American Society of Mechanical Engineers. Proceedings of ASME Turbo Expo 2015, Turbine Technical Conference and Exposition, June 15-19, 2015, Montreal, Canada. New York: ASME, 2015: $18-28$.

[18] DENTON J D. Some limitation of turbomachinery CFD[C]// American Society of Mechanical Engineers. Proceedings of ASME Turbo Expo 2010, Power for Land Sea and Air, June 14-18, 2010, Glasgow, UK. Brussels: ASME, 2010: 735-745.

作者简介: 郑似玉, 女, 1994 年出生。主要研究方向为压气机性能仿真。 E-mail: zhengsiyu@sjtu.edu.cn 滕金芳(通信作者), 女, 1968 年出生, 研究员, 博士研究生导师。主要 研究方向为航空发动机压气机性能模拟、优化及流场测量。

E-mail: tjf@sjtu.edu.cn

美晓青, 男, 1982 年出生, 副研究员, 硕士研究生导师。

E-mail: qiangxiaoqing@sjtu.edu.cn 